encephalopathy. Brain Oct 2010;133(10):2964-2970). (Respond: Eamonn R Meyer, Department of Medical and Molecular Genetics, University of Birmingham School of Medicine, Institute of Biomedical Research, Birmingham, B15 2TT, UK. E-mail: e.r.meyer@bham.ac.uk).

COMMENT. Infantile spasms and West syndrome is a heterogeneous disorder with cryptogenic and symptomatic etiologies. Genetic causes for West syndrome listed by the authors include mutations of ARX, controlling development of g-aminobutyric acid-ergic interneurons, mutations in STXBP1 (in Ohtahara and West syndromes), and deletions involving MAG12. Phospholipase B-b 1 gene is involved in hippocampal muscarinic acetylcholine receptor signaling and in cortical development. The discovery of this mutation uncovers a novel mechanism for early-onset epileptic encephalopathy, infantile spasms and West syndrome.

\title{
EEG AND MR SPECTROSCOPY IN HYPOXIC-ISCHEMIC ENCEPHALOPATHY IN TERM NEWBORNS
}

Researchers from the University of Bologna, Italy, studied the relation of amplitude integrated EEG findings in the first $24 \mathrm{hrs}$ of life to brain metabolic changes, detected by proton MR spectroscopy (H-MRS) at 7-10 days of life, in 32 term newborns with hypoxic-ischemic encephalopathy (HIE). a-EEG at 6, 12 and $24 \mathrm{hrs}$ of life was significantly correlated with outcome, and showed improvement in newborns with normal H-MRS and good outcome and a deterioration in those with abnormal H-MRS and poor outcome. a-EEG time course documents the severity, as defined by H-MRS, and evolution of brain damage following an HI event in non-cooled newborns. Both a-EEG and MRS are correlated with outcome.

At follow-up till 2 years of age in 31 surviving patients, $21(67.7 \%)$ had a normal motor outcome, 7 (22.6\%) developed transitory hypertonia, and $3(9.7 \%)$ developed CP. Newborns with normal motor outcome or transitory hypertonia had a mean General Quotient of 104 on the Griffiths Mental Development Scale. BAER was normal in all cases; a cortical visual impairment occurred in 2 of 3 patients with $\mathrm{CP}$. Eight patients $(25 \%)$ developed neonatal seizures; the a-EEG background was severely abnormal in 3 and moderately abnormal in 5 cases at 6 hrs of life. Newborns with a normal a-EEG background at $6 \mathrm{hrs}$ developed normally, whereas 2 with severely abnormal EEG background had a poor prognosis ( 1 died, 1 had $\mathrm{CP}$ ). Of the 5 with moderately abnormal EEG background pattern at $6 \mathrm{hrs}, 2$ with a deteriorating pattern at $12 \mathrm{hrs}$ developed CP, whereas 3 unchanged at $12 \mathrm{hrs}$ and recovered at $24 \mathrm{hrs}$ showed a normal outcome. Electrographic seizures did not change prognostic accuracy of a-EEG background pattern. MRI abnormalities were observed in 5/31 patients. (Ancora G, Soffritti S, Lodi $\mathrm{R}$, et al. A combined a-EEG and MR spectroscopy study in term newborns with hypoxicischemic encephalopathy. Brain Dev November 2010;32:835-842). (Respond: Dr Gina Ancora, Neonatology Unit, Dept of Woman, Child and Adolescent Health, University of Bologna, Italy. E-mail: gina.ancora@unibo.it).

COMMENT. Seizure activity dedected at a-EEG in HIE newborns was associated with a poor outcome only in patients with abnormal a-EEG background pattern. Patients 
with seizures but normal EEG background at 24 hrs of life had a normal outcome. Background pattern on the a-EEG at onset of seizures or status epilepticus is the best predictor of outcome. (van Rooij LG et al. Pediatrics 2007;120:e354-e363).

H-MRS shows a better correlation with outcome of newborns with HIE compared to conventional MRI. a-EEG has a high sensitivity for alterations in the H-MRS performed in the subacute period after perinatal asphyxia, and both a-EEG and H-MRS have a similar sensitivity and specificity for the prediction of outcome of HIE in term newborn. These a-EEG data obtained at 24 hrs of life may be useful in prediction of subsequent development of seizures in newborns with HIE.

\section{INFANTILE SPASMS CONSENSUS REPORT}

An Infantile Spasms Working Group (ISWG) of 14 pediatric neurologists with expertise in IS participated in a 2-day workshop to discuss 1) the current state of IS management, 2) the evidence for efficacy of ACTH and vigabatrin (VGB), approved in the US in 2009, and need for additional alternatives, and 3) to develop protocols as a guide for the diagnostic workup and management of IS. The incidence of IS ranges from 2 to 3.5 per 10,000 live births, and the lifetime prevalence at 10 years of age is lower at 1.5 to 2 per 10,000 children (Trevathan et al, 1999), a result of high mortality. The ISWG discussed the AAP/AAN/CNS consensus statement and practice guideline of 2004, and reviewed the current literature. A consensus was not reached on initial treatment dosage levels, but consensus was strong for the following conclusions:

1) Need for broad clinical evaluation and diagnostic workup, with overnight inpatient 24-hr video-EEG (awake and all stages of sleep, especially non-REM sleep) conducted as soon as possible after identification of spasms. When EEG hypsarhythmia (Gibbs EL \& FA, 1952, preferred spelling) is documented, MRI and other diagnostic tests should be performed and treatment initiated promptly.

2) ACTH and VGB have proven efficacy and are first-line treatments. VGB is first choice for IS comorbid with tuberous sclerosis, and second or third choice with other symptomatic or cryptogenic IS. Duration of therapy, cumulative dose, and daily dose are implicated as risk factors for visual field changes with VGB; periodic ophthalmic evaluations are recommended in addition to baseline, and at 3-6 months after VGB treatment withdrawal.

3) Timely assessment of treatment efficacy ( 2 weeks for ACTH followed by taper; 2 weeks or less following dose titration for VGB). Longer treatment trials are not likely to be effective and may be associated with serious adverse effects.

4) Response to treatment should be "all or none," with complete cessation of spasms and resolution of hypsarrythmia.

(Pellock JM, Hrachovy R, Shinnar S, Nordli DR, et al. Infantile spasms: a US consensus report. Epilepsia Oct 2010;51(10):2175-2189)(Respond: John M Pellock MD, Children's Pavilion, 1001 East Marshall Street, Richmond, VA 23298. E-mail: jpellock@mcvh.vcu.edu).

COMMENT. In a commentary from Finland on the US consensus report (Riikonen R. Epilepsia 2010;51:2215-6), the dangers of prolonged therapy with either ACTH or VGB are emphasized, and the smallest effective doses for 2 weeks are favored. 